\title{
Atribuições da carne de frango relevantes ao consumidor: foco no bem-estar animal
}

\author{
Anderson Bonamigo ${ }^{1}$, Clarisse Barreto dos Santos Silva Bonamigo ${ }^{2}$, Carla Forte Maiolino Molento ${ }^{1}$ \\ 1 Universidade Federal do Paraná - UFPR. \\ ${ }^{2}$ Agroindústria.
}

RESUMO - Objetivou-se neste trabalho estudar o comportamento dos consumidores de carne de frango em relação ao bem-estar animal e à disposição em pagar mais por produtos com certificação para esse atributo de qualidade. Para isso foi realizada uma pesquisa de mercado com consumidores de carne de frango da cidade de Curitiba, Paraná. Uma análise prévia foi realizada com peritos da cadeia avícola para estruturação do questionário. Após esta fase, 481 consumidores foram entrevistados. Perguntas objetivas resultaram em informações gerais e imagens de produtos hipotéticos geraram informações sobre a atitude de compra utilizando análise conjunta e posterior simulação de mercado. Os atributos mais observados na hora da compra foram validade, preço, cor e odor. O bem-estar animal inicialmente foi considerado pela minoria (3,7\%). Dos entrevistados, 68,5\% não conhecem o sistema de produção, porém, depois de observar fotos dos sistemas, acreditam que o modelo semiintensivo proporciona melhor bem-estar e resulte em produtos de melhor qualidade. Na análise conjunta, preço baixo, produção com melhores condições de bem-estar animal, carne firme e rosada e produção sem antibióticos são, respectivamente, as características com maiores valores de utilidade. O atributo de maior importância foi o preço (34,1\%), seguido do tipo de carne (24,6\%), bem-estar animal (24,1\%) e utilização de antibióticos (17,0\%). Na simulação de mercado, 70,9\% dos consumidores pagariam mais por produtos que com certificação de bem-estar animal e carne firme e rosada. A baixa importância inicial do bem-estar animal para o consumidor pode estar relacionada à falta de conhecimento acerca dos sistemas produtivos, portanto o acesso a informações a respeito pode incentivar o pagamento por esse atributo.

Palavras-chave: atitude de compra, demanda do consumidor, qualidade do produto

\section{Broiler meat characteristics relevant to the consumer: focus on animal welfare}

\begin{abstract}
The objective of this experiment was to study the behavior of chicken consumer, with emphasis on animal welfare and on the willingness to pay a higher value for products with certification for this attribute of quality. Thus, a market research was conducted with consumers of broiler meat in the city of Curitiba, Paraná. A primary analysis was done with experts of the avian chain. After this, 481 consumers were interviewed. Straight questions generated general information and images of the hypothetical products generated information about buying attitudes, using conjoint analysis. The most expressed attributes during the act of buying were shelf-life date, price, color and odor. Animal welfare was considered by a minority of $3.7 \%$. Of the interviewed, $68.5 \%$ did not know the production system, but after having observed photos of the systems, they believed that the semi-intensive system provided higher animal welfare and originated a better product. It was possible to observe that low price, production with better conditions of animal welfare, firm pink meat, and production without antibiotics, respectively, present the highest utility values. The attribute of greater importance was price (34.1\%), followed by the type of meat (24.6\%), animal welfare (24.1\%), and the usage of antibiotics $(17.0 \%)$. The consumers said they would pay more for products that had attributes of animal welfare and firm pink meat. The low initial importance of animal welfare might be related to lack of knowledge of production systems by consumers, and that the improvement in information increases the disposition to pay more for this attribute.
\end{abstract}

Key Words: buying attitude, consumer demanding, quality of the product

\section{Introdução}

Nos últimos anos, a preocupação com a produção de alimentos tem aumentado. Além da crescente demanda provocada pelo aumento da população humana, houve uma alteração dos hábitos alimentares, com crescente preocupação com a qualidade do produto, a saúde e a segurança alimentar (Bernabéu \& Tendero, 2005).

A solução para atender à demanda atual, em quantidade e qualidade suficientes, é desafiadora. Segundo a Food and Agriculture Organization (FAO, 2008), o atual e frágil sistema global de produção de alimentos inevitavelmente 
necessitará de novas tecnologias de produção e intensificação dos atuais modelos. Essa visão gera discussões: Chiu \& Lin (2009) citam que as melhorias nas tecnologias agrícolas não são suficientes para acabar com a fome no mundo, que dietas à base de carne contribuíram para a pressão sobre o sistema alimentar e que uma das soluções seria a substituição desse hábito por uma alimentação vegetal.

Na ética da produção animal, o sofrimento ao qual os animais são expostos nos sistemas de produção intensiva tornou-se um problema evidente. Nos países em que essas preocupações estão presentes, observa-se aumento no número de diretrizes, regras e leis, tanto do setor público quanto do privado, para estabelecimento de padrões mínimos de bem-estar animal (Philips, 2009). Por outro lado, o bem-estar animal tem sido relacionado a aumento no custo de produção (Den Ouden, 1997). Em países em desenvolvimento, questões sobre quem vai pagar pelo aumento do custo das melhorias na qualidade de vida aos animais de produção vem se tornando o principal entrave na comercialização desses produtos (Molento, 2005).

Considerando o maior custo de produção, o aumento dos padrões de bem-estar animal será viável somente quando o consumidor for capaz de perceber a diferença entre produtos e estiver preparado para pagar mais (Verbeke, 2009). Dessa forma, é necessário avaliar constantemente os sinais do mercado consumidor em relação a esse atributo, a fim de medir sua importância para a sociedade em questão.

Este trabalho foi realizado com objetivo de estudar o comportamento dos consumidores de carne de frango em relação ao bem-estar animal e à sua disposição em pagar mais pela carne produzida em sistemas nos quais se considera o bem-estar animal.

\section{Material e Métodos}

Para se conhecer o comportamento do consumidor de carne de frango, foi realizada uma pesquisa com perguntas diretas sobre as preferências e um teste de conceito, utilizando-se pesquisa qualitativa e quantitativa (Kotler, 2000). Este teste de conceito consistiu da apresentação de um produto para um consumidor-alvo e da obtenção de sua resposta para posterior análise. O questionário, previamente submetido e aprovado pelo comitê de ética em pesquisa (Comética-UFPR) sob o registro - CEP/SD: 8811.146.09.10 e CAAE: 0070.0.091.000-09, foi aplicado individualmente para consumidores de Curitiba, Paraná, nos meses de outubro de 2009 a janeiro de 2010 .

Inicialmente, para estruturação do questionário aplicado ao consumidor final, foi realizada uma análise qualitativa para coleta de informações de peritos do setor avícola. Perguntas sobre as preferências do consumidor em relação aos produtos cárneos oferecidos no varejo, seu conhecimento em relação aos sistemas de produção e sobre a relação entre esses sistemas e o custo final do produto foram respondidas por 23 peritos.

A preferência do consumidor, de acordo com os peritos, é pela carne bovina, seguida da carne de frango. Segundo eles, o preço é fator determinante para as classes média e baixa, tornando o frango o principal substituto da carne bovina na hora da aquisição.

Entre as preocupações dos consumidores de carne de frango, foram citadas a utilização de hormônios (citada por $73,9 \%$ dos peritos), a adição de água (47,8\%), a contaminação dos produtos $(26,1 \%)$ e o preço $(17,4 \%)$. Já na hora da compra, as características observadas pelo consumidor são cor, odor, aparência e sabor da carne (Figura 1), dados

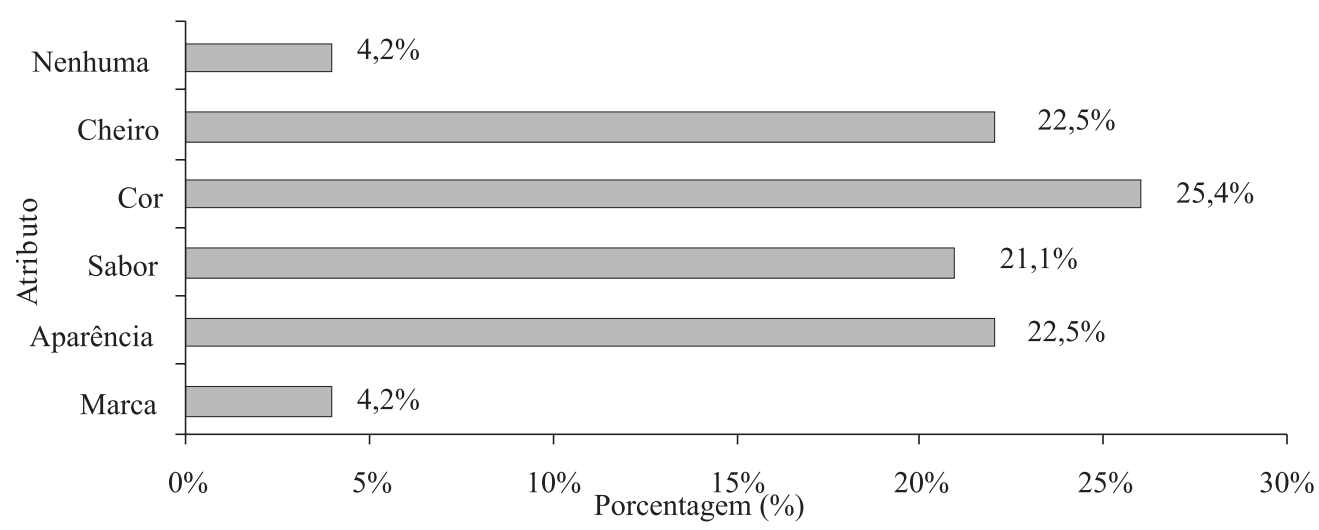

Figura 1 - Características citadas pelos peritos da cadeia avícola como atributos de maior importância para o consumidor na hora da compra. 
semelhantes aos resultados encontrados por Francisco et al. (2007). Conforme os peritos, essas características do produto podem variar entre sistemas de produção. Além da possível diferença no sabor, pode ser observada maior firmeza e maior pigmentação no produto final do sistema semiintensivo. Segundo Zanusso \& Dionell (2003), os principais fatores que interferem nas características organolépticas estão ligados a fatores intrínsecos (idade ao abate, genótipo) e extrínsecos (condições de criação e alimentação) às aves.

As diferenças entre os sistemas produtivos influenciam no custo de produção, que pode ser aproximadamente $75 \%$ maior no semiintensivo, com grande variação entre os modelos adotados. Segundo Rowan et al. (1999), o custo de produção de ovos em sistemas alternativos pode variar entre 35 e $70 \%$. Dessa forma, somente consumidores das classes mais altas pagariam por um produto com maior valor agregado, uma vez que seu conhecimento do consumidor sobre as diferenças dos sistemas de produção é pouco ou nenhum, como citado por $86,9 \%$ dos peritos.

Para a pesquisa quantitativa, foi estipulado o mínimo de 400 entrevistados, determinado por cálculo a partir de erro máximo de 5,0\%, 95,5\% de confiança $(\mathrm{k}=2)$ e princípio máximo de indeterminação $(p=q=50 \%)$. A população amostral seguiu distribuição próxima à da população curitibana em suas nove administrações regionais (Tabela 1), segundo dados publicados pelo Instituto de Pesquisa e Planejamento Urbano de Curitiba (IPPUC, 2005). Respeitando essa divisão regional, os consumidores foram entrevistados ao acaso, em ambientes variados, como parques e terminais rodoviários, entre outros.

A primeira parte do questionário baseou-se em perguntas objetivas, de múltipla escolha, tendo-se como intuito a caracterização dos consumidores locais. Para a segregação por classe social, foram utilizadas questões definidas pelo Critério Brasil (ABEP, 2007), porém

Tabela 1 - Percentual de entrevistas realizadas de acordo com distribuição de pessoas por administrações regionais da cidade de Curitiba, Paraná, nos meses de outubro de 2009 a janeiro de 2010

\begin{tabular}{lc}
\hline Administrações regionais & Percentagem de entrevistas realizadas \\
\hline Matriz & $16,1 \%$ \\
Boqueirão & $13,1 \%$ \\
Cajuru & $10,6 \%$ \\
Boa Vista & $12,1 \%$ \\
Santa Felicidade & $8,5 \%$ \\
Portão & $12,3 \%$ \\
Pinheirinho & $10,0 \%$ \\
Bairro Novo & $7,5 \%$ \\
CIC & $5,8 \%$ \\
Total & $100,0 \%$
\end{tabular}

alterando-se a pergunta da escolaridade do chefe da família pela escolaridade do entrevistado. Além dessas informações, a frequência de consumo de carne de frango, as principais características apreciadas na hora da compra e o grau de conhecimento sobre os sistemas de produção e o bem-estar animal foram temas abordados para construção de uma imagem geral. Para se obter a opinião dos entrevistados sobre as questões de bem-estar, foram apresentadas imagens do modelo de produção intensivo e semi-intensivo.

A segunda parte do questionário foi realizada pela utilização de cartões ilustrados com imagens que representaram produtos cárneos hipotéticos. Esses cartões apresentavam variações de preço, grau de bem-estar animal, utilização de antibióticos promotores de crescimento e aparência da carne, a qual foi representada por variação de cor e textura. Os cartões tiveram como objetivo explorar a opção de compra, relacionando os níveis de preços com a percepção de bemestar animal e aspectos ligados diretamente ao gosto e à saúde do consumidor, separando importância e grau de utilidade de cada atributo e suas variações por meio de análise conjunta. As combinações formadas pelos quatro atributos e seus níveis formaram 24 possibilidades de produtos. Contudo, para evitar fadiga na hora da entrevista, apenas nove combinações foram mantidas (Tabela 2), sendo descartadas aquelas menos prováveis, como, por exemplo, produto com certificação de alto grau de bem-estar animal, produzido sem antibióticos promotores de crescimento combinados com o menor preço.

Os dados obtidos na primeira fase do questionário, associados aos resultados gerados pela análise conjunta, possibilitaram uma simulação com obtenção de uma provável participação de cada um dos produtos no mercado da carne de frango. Os produtos simulados tiveram as seguintes combinações: 1) preço alto, sem adição de antibiótico na ração, carne firme e rosada e sistema de produção ao ar livre; 2) preço baixo, com adição de antibiótico na ração, carne branca e mole e produção industrial; e 3) preço médio, com adição de antibiótico na ração, carne rosada e firme e produção ao ar livre.

Para análise dos resultados, foi utilizada estatística descritiva para as perguntas objetivas e análise conjunta das respostas dos cartões, semelhante ao realizado por Bernabéu \& Tendero (2005).

\section{Resultados e Discussão}

Foram entrevistados 481 indivíduos nas nove regionais administrativas da cidade de Curitiba, Paraná. Nos dados demográficos, as parcelas com maior expressão foram 
Tabela 2 - Produtos hipotéticos utilizados nas entrevistas para estudar o comportamento do consumidor da carne de frango da cidade de Curitiba, meses de outubro de 2009 a janeiro de 2010

\begin{tabular}{|c|c|c|c|c|}
\hline Produto & Variação de preço & Antibiótico na ração & Tipo da carne & Sistema de produção \\
\hline Produto 1 & $\mathrm{R} \$ 3,70$ & Sem & Branca/mole & Industrial \\
\hline Produto 2 & $\mathrm{R} \$ 3,70$ & Com & Rosada/firme & Ar livre \\
\hline Produto 3 & $\mathrm{R} \$ 5,50$ & Com & Branca/mole & Industrial \\
\hline Produto 5 & $\mathrm{R} \$ 5,50$ & Com & Rosada/firme & Ar livre \\
\hline Produto 6 & $\mathrm{R} \$ 5,50$ & Sem & Rosada/firme & Ar livre \\
\hline Produto 7 & $\mathrm{R} \$ 7,40$ & Sem & Rosada/firme & Industrial \\
\hline
\end{tabular}

representadas por pessoas do sexo feminino, 60\%; com 18 a 39 anos de idade, 62\%; casadas, 46\%; com curso superior incompleto ou completo, 62\%; e empregados de terceiros, 46\% (Tabela 3). De acordo com o critério utilizado para segregação dos entrevistados por nível social, a amostra foi

Tabela 3 - Dados demográficos de 481 consumidores de carne de frango entrevistados na cidade de Curitiba, Paraná, nos meses de outubro de 2009 a janeiro de 2010

\begin{tabular}{llc}
\hline Variável & Subdivisões & Percentual de entrevistados \\
\hline Faixa etária & 18 a 29 anos & $31 \%$ \\
& 30 a 39 anos & $31 \%$ \\
& 40 a 49 anos & $19 \%$ \\
& 50 a 59 anos & $11 \%$ \\
Estado Civil & 60 anos ou mais & $8 \%$ \\
& Solteiro & $41 \%$ \\
& Casado & $46 \%$ \\
& Viúvo & $4 \%$ \\
Escolaridade & Divorciado & $8 \%$ \\
& Fundamental incompleto & $3 \%$ \\
& Fundamental completo & $14 \%$ \\
& Médio incompleto & $6 \%$ \\
& Médio completo & $16 \%$ \\
& Superior incompleto & $22 \%$ \\
& Superior completo & $34 \%$ \\
& Pós-graduação & $6 \%$ \\
& Estudante & $15 \%$ \\
& Do lar & $11 \%$ \\
& Profissional liberal & $14 \%$ \\
& Empregado & $46 \%$ \\
& Empregador & $5 \%$ \\
\hline & Aposentado & \\
& & 50
\end{tabular}

composta de 18,4\% de consumidores de nível alto, 49,6\% médio e 32\% de nível baixo.

Em relação ao hábito de consumo de carne, apenas 3,5\% dos entrevistados não consomem carne de frango; 39,1\% consomem esse produto em média duas vezes por semana; $14,2 \%$ consomem três vezes; e 6,8\%, quatro vezes por semana. Somados estes índices, a maior parte da população (60,1\%) consume carne de frango.

Quanto à preferência dos consumidores, 63,9\% optam em primeiro lugar pela carne bovina, seguida da carne de frango, com 20,3\%. Como segunda opção dos consumidores entrevistados, 48,1\% preferem a carne de frango, seguida da carne bovina com 19,1\% (Tabela 4). Essa ordem é semelhante à citada pelos peritos entrevistados nesse trabalho e por Francisco et al. (2007), com a bovina em primeiro lugar, seguida da carne de frango, de peixes e de suínos.

A resposta dos consumidores sobre a opção de compra difere do cenário das preferências, mostrando que 73,4\% dos entrevistados têm como primeira opção de compra a carne bovina e, como segunda opção, com 62,6\% a carne de frango (Tabela 4). A grande participação de mercado da carne bovina e de frango coloca em evidência os hábitos dos consumidores, mostra grande competitividade e caracteriza um mercado com certa elasticidade de preço.

Quando se trata somente da carne de frango, o consumidor cita como quatro principais atributos observados na hora da compra: validade, 74,2\%; preço, 73,4\%; cor, 62,8\%; e odor, 49,4\% (Figura 2). Francisco et al. (2007)

Tabela 4 - Classificação percentual da preferência (Prefere) e da atitude de compra (Compra) dos quatro principais tipos de carnes, 481 consumidores de Curitiba, Paraná, outubro de 2009 a janeiro de 2010

\begin{tabular}{|c|c|c|c|c|c|c|c|c|}
\hline \multirow[t]{3}{*}{ Classificação } & \multicolumn{8}{|c|}{ Tipo de carne } \\
\hline & \multicolumn{2}{|c|}{ Bovino } & \multicolumn{2}{|c|}{ Frango } & \multicolumn{2}{|c|}{ Pescado } & \multicolumn{2}{|c|}{ Suíno } \\
\hline & Prefere (\%) & Compra (\%) & Prefere (\%) & Compra (\%) & Prefere (\%) & Compra (\%) & Prefere (\%) & Compra (\%) \\
\hline $1^{\mathrm{a}}$ & 63,1 & 73,4 & 20,3 & 22,2 & 13,5 & 4,2 & 3,1 & 0,2 \\
\hline $2^{\mathrm{a}}$ & 18,9 & 18,5 & 48,5 & 62,6 & 17,9 & 9,6 & 14,7 & 9,3 \\
\hline $3^{\underline{a}}$ & 13,1 & 4,0 & 22,6 & 12,4 & 31,8 & 39,3 & 32,5 & 44,4 \\
\hline $4^{\underline{\mathrm{a}}}$ & 4,2 & 4,2 & 8,6 & 2,8 & 36,6 & 47 & 50,6 & 46 \\
\hline
\end{tabular}


observaram preocupações semelhantes, sendo a cor e a aparência do produto os dois primeiros atributos observados, seguidos de odor e textura. Dos atributos menos observados entre os quatro principais, foram citados a marca do produto final, com $21,9 \%$, e a qualidade de vida do animal, com 3,7\% dos consumidores que os citaram (Figura 2).

Com relação ao conhecimento do consumidor sobre questões de bem-estar animal, 68,5\% dos entrevistados afirmaram não conhecer os sistemas de produção. Na Europa, parte da população que tem conhecimento sobre as condições da agricultura e do bem-estar animal possui maior tolerância ao aumento do preço desses produtos. Além disso, acredita que o conhecimento e a atitude de compra influenciam na melhoria das condições de bem-estar animal na fase de produção (EUROPEAN COMMISSION, 2007). Assim, o desconhecimento de grande parte dos entrevistados pode explicar a grande diferença entre os atributos preço e bem-estar animal (Figura 2).
Apesar de a maioria dos consumidores entrevistados não conhecer o sistema de produção, ao visualizar imagens dos sistemas, 76,0\% deles atribuíram ao sistema intensivo maior impacto negativo ao bem-estar e, desses, 46,9\% e $29,1 \%$ citaram esse sistema como um modelo ruim ou muito ruim, respectivamente, para o animal. Em contrapartida, quando apresentada uma imagem de frangos com acesso ao pasto, o consumidor associou essa situação a melhor qualidade de vida. Desses, 54,0\% e 37,6\% associaram a imagem do modelo alternativo a qualidade de vida dos frangos boa e muito boa, respectivamente (Figura 3). Na Europa, semelhante aos dados dessa pesquisa, mais de 80\% dos entrevistados avaliaram a situação dos animais de produção como moderadas e muito ruins, sendo que $77 \%$ dos europeus acreditam que é possível se fazer mais para melhorar as condições de bem-estar dos animais (EUROPEANCOMMISSION, 2007).

Neste trabalho, 94,9\% dos consumidores acreditam que o sistema de produção provoca alterações na qualidade

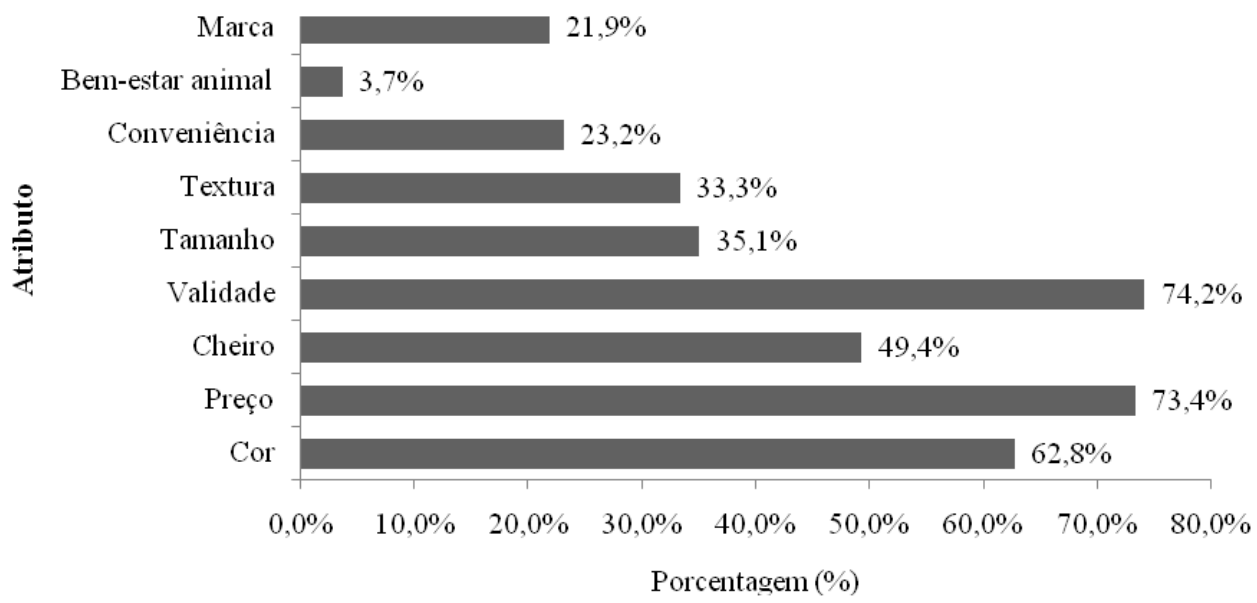

Figura 2 - Percentual de citação de cada característica entre os quatro principais atributos na hora da compra de carne de frango, cidade de Curitiba, Paraná, nos meses de outubro de 2009 a janeiro de 2010.

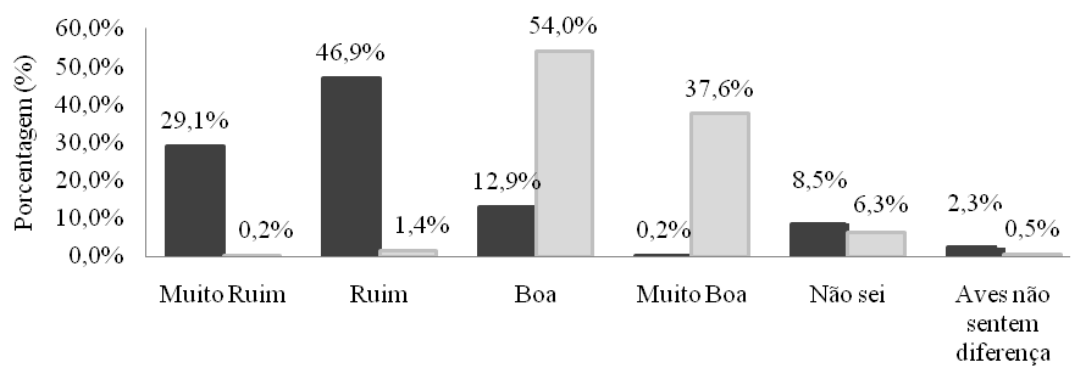

Nível de bem-estar animal

Figura 3 - Visão de 481 consumidores de carne de frango quanto ao grau de bem-estar animal oferecido no sistema de produção intensivo e com acesso ao ar livre, cidade de Curitiba, meses de outubro de 2009 a janeiro de 2010. 
da carne e 88,9\% deles atribuem ao sistema alternativo um produto final de melhor qualidade. Esta possível correlação entre bem-estar e qualidade da carne, segundo a percepção do consumidor, pode representar uma demanda para os dois atributos que se reforçam mutuamente e pode ser utilizada como estratégia para desenvolvimento de produto em determinado segmento de mercado.

Apesar das informações sobre atributos individuais, a opinião do consumidor final é formada por uma combinação de atributos, na qual se deixa de escolher um atributo específico, considerado importante separadamente, em favor da combinação formada. A medida de utilidade de cada um dos atributos nos diferentes níveis oferecidos determinou quanto foi mais desejado pelo consumidor. Uma maior utilidade foi atribuída ao preço de menor valor, seguido de produção com bem-estar animal, tipo de carne rosada e firme e, por fim, produção sem utilização de antibiótico (Tabela 5).

Pela determinação da importância de cada atributo, pode-se observar que o preço continua a ser o fato de maior relevância, com 34,1\% (Figura 4). De acordo com Molento (2005), a baixa renda per capita da população brasileira e inexistência de conhecimento formal sobre bem-estar animal são responsáveis pela opção preferencial a baixos preços, uma vez que a escolha por produtos oriundos de animais criados em condições de maior bem-estar está ligada a uma reflexão profunda sobre o assunto.

Como segundo atributo de maior importância pelos consumidores finais encontram-se o tipo da carne, com $24,6 \%$ e o bem-estar animal com $24,1 \%$ (Figura 4). Esses atributos tiveram importância semelhante, pois os cartões representavam esses elementos de maneira associada. A utilidade observada neste trabalho para esses dois elementos revela a preferência do consumidor por animais produzidos em boas condições de bem-estar animal e que apresentem carne firme e rosada.

Tabela 5 - Estimativa de utilidade dos diferentes níveis dos quatro atributos que compuseram os produtos potenciais oferecidos a 481 consumidores de carne de frango entrevistados na cidade de Curitiba, Paraná, nos meses de outubro de 2009 a janeiro de 2010

\begin{tabular}{llc}
\hline Atributo & \multicolumn{1}{c}{ Nível } & Valor de utilidade \\
\hline Preço & Baixo & 0.7172 \\
& Médio & -0.0402 \\
& Alto & -0.6769 \\
Bem-estar animal & Sem restrições & 0.5644 \\
& Com restrições & -0.5644 \\
Tipo carne & Firme e rosada & 0.3379 \\
& Mole e branca & -0.3379 \\
Tipo de produção & Sem antibiótico & 0.1523 \\
& Com antibiótico & -0.1523 \\
\hline
\end{tabular}

A utilização de antibióticos na fase de produção foi o atributo de menor importância, com 17,0\%. Ao contrário desse resultado, percebe-se no mundo forte preocupação com a segurança alimentar. Cowan (2007) observou forte preocupação entre consumidores europeus e, em relação à carne de frango, salmonela, hormônios e antibióticos foram os mais citados. Nos Estados Unidos, há um crescente aumento na demanda de produtos orgânico certificados pelo governo, os quais são livres de antibióticos e com menores restrições de bem-estar, atributos interligados na hora da compra (Husak et al., 2008). A maior preocupação com a utilização de antibióticos na produção, principalmente nos países europeus, pode ser reflexo das diferenças históricas. $\mathrm{Na}$ Europa, essas preocupações foram originadas já em 1969, quando houve, na Grã-Bretanha, o primeiro questionamento sobre a utilização de tetraciclinas e penicilinas como promotores de crescimento e o aparecimento de Salmonella typhimurium resistente (Barton, 2000). De maneira similar às questões de bem-estar, também pode haver dificuldade de posicionamento por desinformação da população entrevistada.

$\mathrm{Na}$ análise de participação de mercado, quando comparados os três produtos inicialmente propostos, observou-se participação semelhante, com maior frequência nos produtos de carne rosada/firme e proveniente de sistema de produção com acesso ao ar livre (Tabela 6). Este fato está relacionado diretamente ao alto valor relativo obtido da soma desses dois atributos, equilibrando, assim, a maior importância e utilidade atribuída pelo consumidor ao menor preço. Pode-se supor neste trabalho que o uso de cartões que simbolizavam os produtos hipotéticos, os quais possuíam ilustrações de cada atributo, foi estimulada a reflexão do consumidor sobre cada um deles. Em consequência, o impacto da imagem de animais em melhores condições de bem-estar pode ter sido determinante para o resultado desta análise.

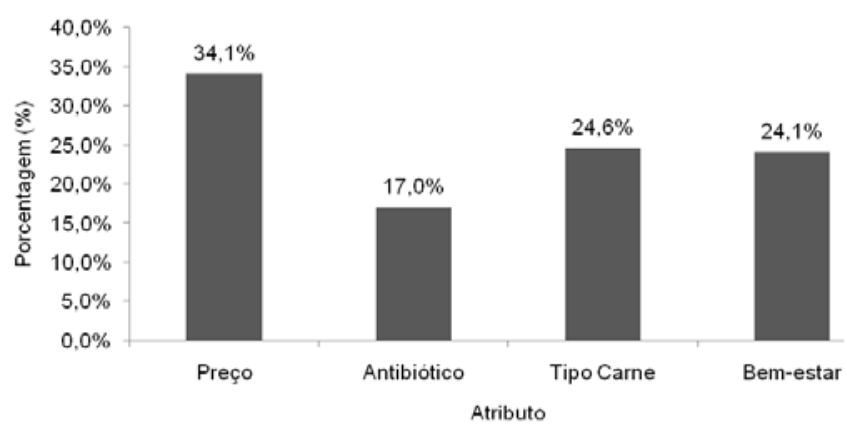

Figura 4 - Estimativa de importância dos quatro atributos que compuseram os produtos potenciais oferecidos a 481 consumidores de carne de frango, entrevistados na cidade de Curitiba, Paraná, nos meses de outubro de 2009 a janeiro de 2010 . 
Tabela 6 - Simulação da participação de mercado de três diferentes produtos, obtida por valores de utilidade de cada atributo segregados pelo nível social de 481 consumidores entrevistados na cidade de Curitiba no período de outubro de 2009 a janeiro de 2010

\begin{tabular}{|c|c|c|c|c|c|c|}
\hline \multirow[t]{3}{*}{ Nível social } & \multicolumn{6}{|c|}{ Tipo do produto } \\
\hline & \multicolumn{2}{|c|}{$\begin{array}{l}\text { Preço alto, sem antibiótico } \\
\text { na ração, carne rosada e firme } \\
\text { e sistema com acesso ao ar livre }\end{array}$} & \multicolumn{2}{|c|}{$\begin{array}{l}\text { Preço baixo, com antibiótico } \\
\text { na ração, carne branca e mole } \\
\text { e sistema industrial }\end{array}$} & \multicolumn{2}{|c|}{$\begin{array}{l}\text { Preço médio, com antibiótic } \\
\text { na ração, carne rosada e firm } \\
\text { e sistema com acesso ao ar livr }\end{array}$} \\
\hline & $(\%)$ & $\mathrm{n}$ & $(\%)$ & $\mathrm{n}$ & $(\%)$ & $\mathrm{n}$ \\
\hline Alta & $32.5 \%$ & 25 & $33.8 \%$ & 26 & $33.8 \%$ & 26 \\
\hline Média & $29.8 \%$ & 62 & $29.3 \%$ & 61 & $40.9 \%$ & 85 \\
\hline Baixa & $38.8 \%$ & 52 & $26.1 \%$ & 35 & $35.1 \%$ & 47 \\
\hline População geral & $33.2 \%$ & 139 & $29.1 \%$ & 122 & $37.7 \%$ & 158 \\
\hline
\end{tabular}

\section{Conclusões}

O preço da carne de frango é o atributo de maior importância para o consumidor na hora da compra. Inicialmente, o bem-estar animal não é um atributo importante, e isso pode ser explicado parcialmente pela falta de informação do consumidor sobre os modelos de produção adotados. Quando uma reflexão sobre o bem-estar animal é estimulada, a maioria dos consumidores mostra-se disposta a pagar um valor adicional pelo produto com tal atributo, combinado com a característica carne mais firme e rosada. O aumento da qualidade de vida dos animais depende de ações voltadas ao consumidor final. Entre as ações iniciais, faz-se fundamental a divulgação de informações claras e confiáveis sobre o sistema de produção adotado e seus impactos no bem-estar animal.

\section{Referências}

ASSOCIAÇÃO BRASILEIRA DAS EMPRESAS DE PESQUISA ABEP. [2007]. Critério de classificação econômica Brasil. Disponível em: <http://www.abep.org/novo/CMS/Utils/FileGenerate. ashx?id=12> Acesso em: 20 mar. 2009.

BARTON, M.D. Antibiotic use in animal feed and its impact on human healt. Nutrition Research Reviecws, v.13, p.279-299, 2000.

BERNABÉU, R.; TENDERO, A. Preference structure for lamb meat consumers. A Spanish case study. Meat Science, v.71, p.464470, 2005.

CHIU, T.H.T.; LIN, C.L. Ethical management of food systems: plant based diet as a holist approach. Asia Pacific Journal of Clinical Nutrition, v.18, p.647-653, 2009.

COWAN, C. Irish and European consumers view on food safety. Journal of Food Safety, v.18, p.275-299, 2007.

DEN OUDEN, M.; NIJSING, J.T.; DIJKHUIZEN, A.A. et al. Economic optimization of pork production-marketing chains:
I. Model input on animal welfare and costs. Livestock Production Science, v.48, p.23-37, 1997

EUROPEAN COMMISSION [2007] Attitudes of EU citizens towards animal welfare. Office for Official Publications of the European Communities: Luxembourg, 2007. Disponível em: $<$ http://ec.europa.eu/public_opinion/archives/ebs/ ebs_295_en.pdf>. Acesso em: 20 jan. 2010.

FOOD AND AGRICULTURE ORGANIZATION - FAO. How to feed the world in 2050. Disponível em: <http://www.fao.org/fileadmin/ templates/wsfs/docs/expert_paper/How_to_ Feed_the_World_in_ 2050_01.pdf> Acesso em: 8 out. 2009.

FRANCISCO, D.C.; NASCIMENTO, V.P.; LOGUERCIO, A.P. et al. Caracterização do consumidor de carne de frango da cidade de Porto Alegre. Ciências Rurais, v.37, p.253-258, 2007.

HUSAK, R.L.; SEBRANEK, J.G.; BREGENDAHL, K.A survey of commercially available broilers marketed as organic, free-range, and conventional broilers for cooked meat yields meat composition, and relative value. Poultry Science, v.87, p.2367-2376, 2008.

INSTITUTO DE PESQUISA E PLANEJAMENTO URBANO DE CURITIBA - IPPUC [2005]. Estimativa de população e densidade e área das administrações regionais e respectivos bairro. Disponível em: <http://ippucnet.ippuc.org.br/Bancodedados/ Curitibaemdados/anexos/ 2005_Estimativas\%20de\%20Popula\% C3\%A7\%C3\%A3o,\%20\%C3\%81rea\%20e\%20Densidade,\% 20Regionais\%20e\%20Bairros\%20de\%20Curitiba.pdf.> Acesso em: 1 out. 2008.

KOTLER, P. Administração de marketing. 10.ed. São Paulo: Prentice Hall, 2000. 765p.

MOLENTO, C.F.M. Bem-estar e produção animal: aspectos econômicos - revisão. Archives of Veterinary Science, v.9, n1, p.1-11, 2005.

PHILLIPS, C. The welfare of animal: the silent majority. Neatherland: Springer, 2009. 220p.

ROWAN, A.N.; O'BRIEN, H.; THAYER, L. et al. Farm animal welfare - the focus of animal protection in the USA in the 21st century. Massachusetts: Tufts Center for Animals and Public Policy, 1999. 99p.

VERBEKE, W. Stakeholder, citizen and consumer interests in farm animal welfare. Animal Welfare, v.18 p.325-333, 2009.

ZANUSSO, J.T.; DIONELLO, N.J.L. Produção avícola alternativa - Análise dos fatores qualitativos da carne de frango de corte tipo caipira. Revista Brasileira de Agrociência, v.9, n3, p.191-194, 2003. 